\title{
Self-perceived Fracture Risk in the Global Longitudinal Study of Osteoporosis in Women: Its Correlates and Relationship with Bone Microarchitecture
}

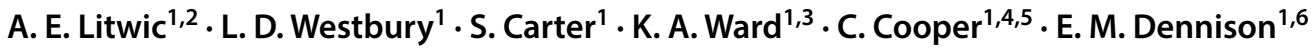

Received: 6 December 2019 / Accepted: 21 February 2020 / Published online: 5 March 2020

(c) The Author(s) 2020

\begin{abstract}
The purpose of this study is to examine correlates of self-perceived fracture risk (SPR) and relationships between SPR and subsequent bone density and microarchitecture in the UK arm of the Global Longitudinal Study of Osteoporosis in Women. 3912 women completed baseline questionnaires detailing medical history and SPR; 492 underwent HRpQCT scans of the radius and tibia and DXA scans of total body, hip, femoral neck and lumbar spine a median of 7.5 years later. Correlates of SPR were examined and a cluster analysis of potential predictors of SPR performed. SPR in relation to HRpQCT and aBMD parameters was examined using linear regression with and without adjustment for anthropometric, demographic and lifestyle covariates. Mean (SD) baseline age was 69.0 (9.0) years; 56.6\% reported a similar SPR; $28.6 \%$ lower SPR; $14.9 \%$ higher SPR compared to women of similar age. In mutually-adjusted analysis, higher SPR was associated $(p<0.05)$ with: lower physical activity and educational attainment; use of anti-osteoporosis medications (AOM) and calcium supplements; greater number of falls in the previous year; history of fracture since aged 45; family history of hip fracture; and increased comorbidity. Higher SPR, history of fracture, and use of AOM, calcium and vitamin D clustered together. Even after adjustments that included AOM use, higher SPR was associated with: lower radial trabecular volumetric density and number, and higher trabecular separation; lower tibial cortical area and trabecular volumetric density; and lower aBMD at the femoral neck. Despite greater AOM use, women with higher baseline SPR had poorer subsequent bone health.
\end{abstract}

Keywords Self-perceived fracture risk $\cdot$ Determinants $\cdot$ HRpQCT $\cdot$ DXA $\cdot$ Osteoporosis $\cdot$ Epidemiology $\cdot$ Fracture risk assessment

Electronic supplementary material The online version of this article (https://doi.org/10.1007/s00223-020-00680-9) contains supplementary material, which is available to authorized users.

\section{E. M. Dennison}

emd@mrc.soton.ac.uk

1 MRC Lifecourse Epidemiology Unit, University of Southampton, Southampton, UK

2 Department of Nephrology, Transplantology and Internal Medicine, Medical University of Gdańsk, Gdańsk, Poland

3 MRC Nutrition and Bone Health Research Group, Cambridge, UK

4 NIHR Southampton Biomedical Research Centre, University of Southampton and University Hospital Southampton NHS Foundation Trust, Southampton, UK

5 NIHR Oxford Biomedical Research Centre, University of Oxford, Oxford, UK

6 Victoria University of Wellington, Wellington, New Zealand

\section{Introduction}

Osteoporosis, a disease characterised by low bone mass and structural deterioration, is classified as a public health problem due to its association with an increased risk for fragility fractures and, consequently has a high impact on quality of life and high rates of morbidity [1]. Worldwide, there are nearly nine million osteoporotic fractures each year, with reports suggesting that one in two women and one in five men will experience a fracture in their remaining lifetime from the age of 50 years [1,2]. With ageing of the population, the economic cost of osteoporosis and fractures is projected to increase in the EU from $€ 37.4$ billion in 2010 to $€ 46.8$ billion by 2025 and, in the US, from $\$ 17$ billion in 2005 to $\$ 25.3$ billion by $2025[3,4]$.

Patient and healthcare provider awareness of individual fracture risk is essential for accurate planning and successful implementation of prevention strategies. A number of 
web-based tools have been developed to improve the identification of individuals at high fracture risk. Clinical risk factors such as age, weight and skeletal properties are included in fracture prediction algorithms, with the most commonly used globally being FRAX. Recently, it has been reported in the Global Longitudinal Study of Osteoporosis in Women (GLOW) that self-perceived fracture risk (SPR) may also capture aspects of fracture risk not measured using current risk prediction tools, and has been associated with fracture risk independently of FRAX [5].

Self-perception of risk of a condition is a difficult concept, as it requires an individual to compare their own health status to others. There is evidence that self-perception of risk of osteoporosis and osteoporotic fractures is underestimated in postmenopausal women worldwide [6], and that self-perceived risks of osteoporosis and fracture affect certain behaviours such as seeking medical advice, anti-osteoporosis medication use and BMD screening, which might lead to greater healthcare engagement, treatment and altered bone health [5, 7]. Furthermore, findings from the GLOW cohort suggest that increased self-perceived fracture risk is strongly associated with incident fracture rate [8]. However, very little is known about what determines self-perceived fracture risk.

To address this, we have used data from the UK arm of the GLOW to: identify correlates of SPR; examine how these correlates interrelate by performing a cluster analysis; and relate SPR to subsequent bone density and microarchitecture.

\section{Methods}

\section{Study Participants}

GLOW is a prospective, observational cohort study conducted through general physician practices in 10 countries. Study design and recruitment have been described in detail previously [9]. In brief, practices, representative of each region, were recruited through primary care networks and provided the names of women aged 55 years and older who had been seen by their physician in the past 24 months. The primary aim of GLOW was to characterise the descriptive epidemiology and health impact of osteoporosis-related fractures among women who were 55 years of age and older worldwide. Globally, GLOW enrolled over 60,000 women through over 700 physicians in 10 countries, and conducted annual follow-up for up to 5 years. In Southampton only, a subgroup of participants with baseline data and at least one follow-up questionnaire were invited, after completion of 5 years of follow-up, for a follow-up study which included dual-energy X-ray absorptiometry (DXA) and high resolution peripheral quantitative computed tomography
(HRpQCT) scans. Participants were scanned between April 2014 and December 2017. Patients, who were institutionalized or were not able to complete the study survey by themselves due to cognitive impairment, language barriers, institutionalization, or were too ill to complete the survey or attend for the scans were excluded.

\section{Baseline Questionnaires}

To ascertain self-perceived fracture risk (SPR), participants were asked to rate their risk of fracturing/breaking a bone, compared to other women of the same age, out of the following responses: 'much lower'; 'a little lower'; 'about the same'; 'a little higher'; and 'much higher'. Fracture history since age 45 years was ascertained at the following locations: clavicle, upper arm, wrist, spine, rib, hip, pelvis, ankle, upper leg and lower leg. Family history of hip fracture was obtained by asking participants whether their mother or father had ever broken or fractured their hip. Information on the number of falls during the previous 12 months was also collected.

Further information ascertained from questionnaires included: age; self-reported height and weight; smoking status; alcohol consumption; physical activity; educational attainment; current use of anti-osteoporotic medication (AOM), calcium supplements and Vitamin D supplements (or multivitamin with Vitamin D); current/previous use of oestrogen or hormone replacement therapy (HRT); and years since menopause. Participants were considered to be taking AOM if they reported current use of alendronate, calcitonin, etidronate, ibandronate, pamidronate, raloxifene, risedronate, strontium ranelate, teriparatide, tibolone or zoledronic acid. Participants were asked whether a doctor or health provider had ever told them that they had the following conditions: hypertension; heart disease; high cholesterol; asthma; chronic bronchitis/emphysema; osteoporosis; osteoarthritis/degenerative joint disease; rheumatoid arthritis; stroke; ulcerative colitis/Crohn's disease; celiac disease; Parkinson's disease; multiple sclerosis; cancer; and type 1 diabetes.

\section{Anthropometry and DXA}

In a subgroup of participants that underwent DXA at a median (lower quartile, upper quartile) of $7.5(7.1,8.9)$ years after the baseline questionnaire, height was measured to the nearest $0.1 \mathrm{~cm}$ using a Marsden stadiometer on the day of scanning; weight was measured to the nearest $0.1 \mathrm{~kg}$ using a Marsden MPPS-250 (Marsden Weighing Machine Group Limited, Rotherham, UK) digital floor scale. Areal bone mineral density $\left(\mathrm{aBMD}, \mathrm{g} / \mathrm{cm}^{2}\right.$ ) of the total body, hip, femoral neck and lumbar spine was measured using a DXA 
Hologic Horizon W (software version Apex 5.5.3.1 [Vertec Scientific, Reading, UK]).

\section{Assessment of Bone by HRpQCT}

This subgroup of participants also underwent a HRpQCT scan of the non-dominant distal radius and tibia using XtremeCT I (Scanco Medical, Basserdorf, Switzerland) on the same day as the DXA scan; if there was a history of fracture on the non-dominant limb, the non-fractured limb was measured. A stack of 104 parallel HRpQCT slices were acquired with an isotropic voxel size of $82 \mu \mathrm{m}$. Methods used to process the HRpQCT data have been described previously [10]. For this analysis, the standard evaluation and cortical porosity scripts were run to obtain estimates of the following parameters at the radius and tibia: total area and trabecular area, volumetric density, number, thickness and separation; cortical area, thickness, volumetric density and pores diameter; and cortical porosity [11].

\section{Derived Variables}

Self-reported body mass index (BMI) at baseline was calculated from the self-reported measures of height and weight. Self-reported height and weight were correlated $(r=0.32$, $p<0.001$ ); a sex-specific standardised residual of weightadjusted-for-height at baseline was derived as a marker of adiposity for inclusion in regression models. Variables for BMI and weight-for-height residual were also calculated at follow-up from measured height and weight among the subgroup that underwent DXA and HRpQCT. The total number of comorbidities at baseline, excluding osteoporosis, was used as a marker for overall morbidity. FRAX scores for 10-year probability of major osteoporotic fracture (MOF) and hip fracture were calculated for women from their baseline survey responses, without inclusion of bone mineral density measurements.

\section{Statistical Analysis: Cross-Sectional Correlates of SPR at Baseline}

Participant characteristics of the 3912 women with data on SPR at baseline were described using summary statistics (Table 1). Ordinal logistic regression was used to examine univariate associations between participant characteristics and SPR. Characteristics significantly associated $(p<0.05)$ with SPR were then included in a mutually-adjusted model; FRAX scores were not included in mutually-adjusted analyses as the inclusion of these variables and participant characteristics which are components of FRAX may result in multicollinearity. Sensitivity analyses were performed among the following groups; have osteoporosis; current use of AOM; have osteoporosis or current use of AOM.

\section{Statistical Analysis: Cluster Analysis of Potential Predictors of SPR}

A cluster analysis of the participant characteristics in Table 1 (excluding SPR, osteoporosis and FRAX probability variables and only using self-reported height and weight-for-height residual as measures of anthropometry) was performed among the 2582 participants with complete data on these characteristics; a flow diagram for the various samples of participants used for analysis is presented in Fig. 1. This used the TwoStep Cluster Analysis procedure in SPSS (version 25) which is suitable for a mixture of categorical and continuous variables [12]. This procedure involves grouping observations into clusters based on the distance measure and then applying a hierarchical clustering algorithm to these clusters; the cluster solution with the lowest Bayesian information criterion (BIC) is selected as optimal. The change in log-likelihood from merging two clusters as opposed to keeping them separate was used as the distance measure. Goodness-of-fit of the cluster solution was determined using the silhouette coefficient, a measure of how similar participants are within clusters compared to how similar they are between clusters, which ranges from -1 to $1(<0.2$ : poor; $0.2-0.5$ : fair; >0.5: good). Participant characteristics were then compared between the clusters using descriptive statistics.

\section{Statistical Analysis: SPR in Relation to DXA aBMD and HRpQCT Parameters}

The sample for this subgroup analysis comprised 492 individuals with data on SPR and at least one of the HRpQCT parameters outlined above. Of these 492 participants, 384 and 477 had data on at least one radial and tibial HRpQCT parameter, respectively; the number of participants with available data for the DXA aBMD parameters ranged from 410 to 471 , depending on the parameter (Fig. 1). Participant characteristics of this whole subgroup were described using summary statistics. Linear regression was used to examine SPR in relation to the HRpQCT parameters of the tibia and radius and the aBMD parameters. Unadjusted and adjusted associations, accounting for age at time of scan, follow-up time, measured height at follow-up, weightfor-height residual from measured values at follow-up, physical activity, smoking status, alcohol consumption, education, time since last menstrual cycle, use of AOM, calcium and vitamin D supplements, and oestrogen/HRT, were examined. SPR was treated as an ordinal variable 
Table 1 Baseline participant characteristics of the analysis sample $(n=3912)$

\begin{tabular}{|c|c|c|}
\hline Participant characteristic & $N(\%)$ & Missing values \\
\hline Age (years)* & $69.0(9.0)$ & 0 \\
\hline Self-reported height $(\mathrm{cm})^{*}$ & $161.7(6.8)$ & 193 \\
\hline Self-reported weight $(\mathrm{kg})^{*}$ & $68.3(12.8)$ & 215 \\
\hline $\operatorname{BMI}\left(\mathrm{kg} / \mathrm{m}^{2}\right)^{*}$ & $26.1(4.7)$ & 354 \\
\hline Current smoker & $273(7.1 \%)$ & 48 \\
\hline \multicolumn{3}{|l|}{ Self-perceived fracture risk } \\
\hline Much lower & $472(12.1 \%)$ & 0 \\
\hline A little lower & $646(16.5 \%)$ & \\
\hline About the same & $2213(56.6 \%)$ & \\
\hline A little higher & $442(11.3 \%)$ & \\
\hline Much higher & $139(3.6 \%)$ & \\
\hline \multicolumn{3}{|l|}{ Alcohol consumption } \\
\hline None & $1242(32.0 \%)$ & 34 \\
\hline $1-6$ & $1598(41.2 \%)$ & \\
\hline $7-13$ & $779(20.1 \%)$ & \\
\hline $14-20$ & $222(5.7 \%)$ & \\
\hline$>20$ & $37(1.0 \%)$ & \\
\hline \multicolumn{3}{|l|}{ Physically active compared to others } \\
\hline Not at all & $135(3.5 \%)$ & 56 \\
\hline A little & $694(18.0 \%)$ & \\
\hline Somewhat & $1893(49.1 \%)$ & \\
\hline Very & $1134(29.4 \%)$ & \\
\hline \multicolumn{3}{|l|}{ Educational attainment } \\
\hline Below GCSE & $1540(39.4 \%)$ & 0 \\
\hline GCSE & $1185(30.3 \%)$ & \\
\hline A-level & $522(13.3 \%)$ & \\
\hline Degree & $665(17.0 \%)$ & \\
\hline Current use of anti-osteoporotic medication & $348(9.4 \%)$ & 222 \\
\hline Ever used oestrogen/hormone replacement therapy & $1328(34.6 \%)$ & 71 \\
\hline Currently taking calcium & $736(19.3 \%)$ & 97 \\
\hline Currently taking Vit D/multivitamin with Vit D & $695(18.2 \%)$ & 103 \\
\hline \multicolumn{3}{|l|}{ Years since menopause } \\
\hline Less than 10 years & $677(17.8 \%)$ & 98 \\
\hline $10-19$ years & $1195(31.3 \%)$ & \\
\hline $20-29$ years & $1050(27.5 \%)$ & \\
\hline 30 or more years & $892(23.4 \%)$ & \\
\hline \multicolumn{3}{|l|}{ Falls in previous 12 months } \\
\hline None & $2394(61.9 \%)$ & 44 \\
\hline Once & $902(23.3 \%)$ & \\
\hline 2 times or more & $572(14.8 \%)$ & \\
\hline Fracture since 45 years & $763(20.5 \%)$ & 182 \\
\hline Family history of hip fracture & $490(14.3 \%)$ & 489 \\
\hline FRAX 10-year probability $(\mathrm{MOF})^{\dagger}$ & $10.9(7.3,17.6)$ & 1359 \\
\hline FRAX 10-year probability (hip fracture) ${ }^{\dagger}$ & $2.2(1.1,5.9)$ & 1359 \\
\hline Osteoporosis & $413(10.9 \%)$ & 138 \\
\hline \multicolumn{3}{|l|}{ Number of comorbidities } \\
\hline 0 & $840(24.9 \%)$ & 543 \\
\hline 1 & $1002(29.7 \%)$ & \\
\hline 2 & $826(24.5 \%)$ & \\
\hline 3 & $445(13.2 \%)$ & \\
\hline 4 or more & $256(7.6 \%)$ & \\
\hline
\end{tabular}

*Mean (SD), ${ }^{\dagger}$ Median (lower quartile, upper quartile)

$M O F$ major osteoporotic fracture 
Fig. 1 Flow diagram for the analytical samples of participants

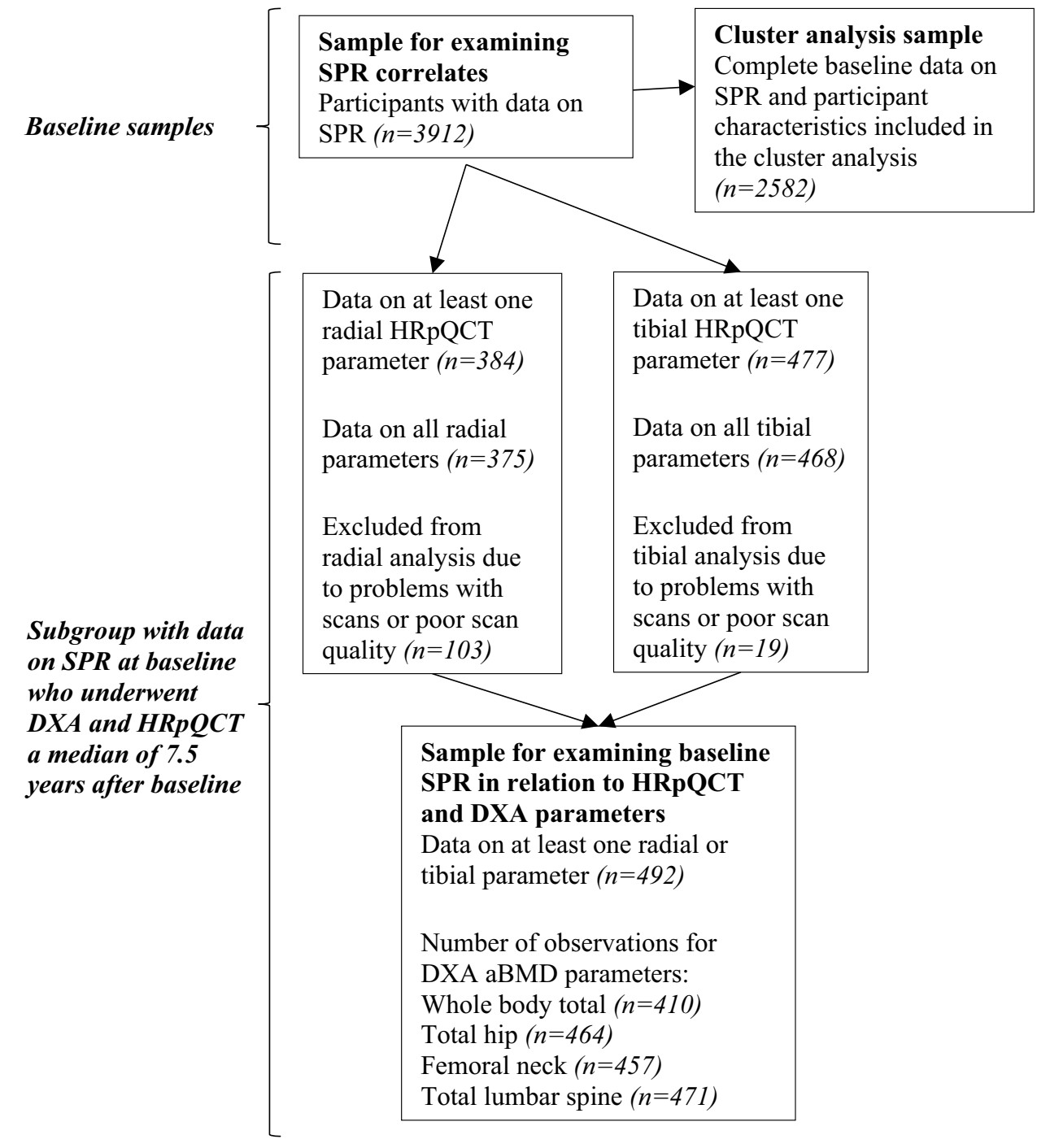

SPR: Self-perceived fracture risk; DXA: Dual-energy X-ray absorptiometry; aBMD: Areal bone mineral density; HRpQCT: High resolution peripheral quantitative computed tomography with five levels. Apart from the cluster analysis, all analyses were conducted using Stata, version 15.0

\section{Results}

\section{Participant Characteristics}

Baseline participant characteristics of the baseline analysis sample $(n=3912)$ are presented in Table 1. Mean (SD) age was 69.0 (9.0) years. Overall, $2213(56.6 \%)$ reported a similar SPR compared to other women of the same age; 1118 (28.6\%) reported a lower risk and 581 (14.9\%) reported a higher risk. Median (lower quartile, upper quartile) FRAX probabilities for 10-year MOF and hip fracture are presented in Table 1. MOF FRAX probabilities for women with lower, similar and higher SPR were 10.4 (7.1, 16.0), 10.7 (7.1, 17.2) and $15.6(9.1,22.8)$, respectively; corresponding FRAX probabilities for hip fracture were $2.1(1.1,5.4), 2.1(1.0,5.5)$ and $3.7(1.6,8.8)$ (data not shown).

Participant characteristics for the subgroup analysis sample $(n=492)$ who underwent bone assessments are presented in Table 2. Mean (SD) age at scan was 70.9 (5.4) years, resulting in a median (lower quartile, upper quartile) follow-up time of $7.5(7.1,8.9)$ years. Overall, $283(57.5 \%)$ reported a similar SPR compared to other women of the same age; $140(28.5 \%)$ reported a lower risk and $69(14.0 \%)$ reported a higher risk. 
Table 2 Baseline characteristics of subgroup who participated in bone phenotyping study $(n=492)$
Participant characteristic

$N(\%)$

Non-missing values

Age of SPR ascertainment*

$$
63.0(5.4)
$$

492

Age at scan (years)*

$70.9(5.4)$

489

Height $(\mathrm{cm})^{*}$

Weight $(\mathrm{kg}) *$

$160.3(6.2)$

482

$68.7(12.7)$

482

$26.8(5.0)$

482

Whole body total aBMD $\left(\mathrm{g} / \mathrm{cm}^{2}\right)^{*}$

$1.01(0.10)$

410

$0.84(0.11)$

464

$0.69(0.10)$

457

$0.92(0.15)$

471

$69(14.4 \%)$

478

$63(14.3 \%)$

442

Family history of hip fracture

$42(8.5 \%)$

492

Much lower

$98(19.9 \%)$

$283(57.5 \%)$

$58(11.8 \%)$

$11(2.2 \%)$

Much higher

How active compared to others

Not at all

$7(1.4 \%)$

A little

Somewhat

$69(14.2 \%)$

$241(49.5 \%)$

Very

Current smoker

$170(34.9 \%)$

$28(5.7 \%)$

Alcoholic drinks per week

None

$101(20.6 \%)$

490

1-6

$208(42.4 \%)$

7-13

14-20

$131(26.7 \%)$

$39(8.0 \%)$

$11(2.2 \%)$

Education

Below GCSE

$120(24.4 \%)$

492

CSE O level/GCSE

$170(34.6 \%)$

A-level

$61(12.4 \%)$

Degree

$141(28.7 \%)$

$31(6.5 \%)$

478

Use of anti-osteoporotic medication

$101(20.9 \%)$

484

$112(23.2 \%)$

482

Currently taking Vit D/multivitamin with Vit D

$238(48.6 \%)$

490

Years since last menstrual cycle

$$
<10
$$

$153(31.9 \%)$

479

$212(44.3 \%)$

$89(18.6 \%)$

20-29

$25(5.2 \%)$

$>29$

*Mean (SD)

$S P R$ self-perceived fracture risk, $D X A$ dual-energy X-ray absorptiometry, $a B M D$ areal bone mineral density 
Table 3 Odds ratios for having a higher category of self-perceived fracture risk for the presence versus absence of each characteristic

\begin{tabular}{|c|c|c|c|c|}
\hline \multirow[t]{2}{*}{ Characteristic } & \multicolumn{2}{|l|}{ Univariate } & \multicolumn{2}{|l|}{ Mutually-adjusted } \\
\hline & Odds ratio $(95 \% \mathrm{CI})$ & $p$-value & Odds ratio $(95 \% \mathrm{CI})$ & $p$-value \\
\hline Age* & $1.02(0.96,1.08)$ & 0.584 & & \\
\hline Self-reported height* & $0.92(0.86,0.97)$ & 0.006 & $0.95(0.87,1.02)$ & 0.152 \\
\hline Weight-for-height residual* & $1.02(0.96,1.09)$ & 0.527 & & \\
\hline Current smoker & $1.11(0.88,1.41)$ & 0.388 & & \\
\hline Alcohol consumption** & $0.94(0.88,1.00)$ & 0.049 & $1.04(0.95,1.13)$ & 0.399 \\
\hline Physically active compared to others of similar age** & $0.52(0.48,0.57)$ & $<0.001$ & $0.52(0.47,0.58)$ & $<0.001$ \\
\hline Educational attainment ${ }^{* *}$ & $0.90(0.85,0.95)$ & $<0.001$ & $0.87(0.81,0.93)$ & $<0.001$ \\
\hline Current use of anti-osteoporotic medication & $8.99(7.15,11.29)$ & $<0.001$ & $6.10(4.48,8.32)$ & $<0.001$ \\
\hline Ever used oestrogen/hormone replacement therapy & $1.06(0.94,1.21)$ & 0.345 & & \\
\hline Currently taking calcium supplements & $3.04(2.58,3.59)$ & $<0.001$ & $1.64(1.32,2.03)$ & $<0.001$ \\
\hline Currently taking Vit D/multivitamin with Vit D & $1.09(0.93,1.28)$ & 0.300 & & \\
\hline Years since menopause ${ }^{* *}$ & $1.06(1.00,1.13)$ & 0.040 & $0.82(0.76,0.89)$ & $<0.001$ \\
\hline Falls in previous 12 months $* *$ & $1.44(1.32,1.57)$ & $<0.001$ & $1.23(1.10,1.37)$ & $<0.001$ \\
\hline Fracture since 45 years & $3.49(2.96,4.12)$ & $<0.001$ & $2.63(2.13,3.24)$ & $<0.001$ \\
\hline Family history of hip fracture & $1.34(1.12,1.62)$ & 0.002 & $1.40(1.13,1.74)$ & 0.002 \\
\hline FRAX 10-year probability (MOF)* & $1.26(1.16,1.36)$ & $<0.001$ & & \\
\hline FRAX 10-year probability (hip fracture)* & $1.18(1.09,1.27)$ & $<0.001$ & & \\
\hline Number of comorbidities $* *$ & $1.20(1.13,1.27)$ & $<0.001$ & $1.08(1.01,1.15)$ & $\mathbf{0 . 0 3 3}$ \\
\hline
\end{tabular}

Ordinal logistic regression models were used with the 5-level variable for self-perceived fracture risk as the outcome

Significant associations $(p<0.05)$ are highlighted in bold. All characteristics were ascertained at baseline

* Odds ratio per standard deviation increase

** Odds ratio per higher category of characteristic

\section{Associations Between Baseline Participant Characteristics and SPR}

Cross-sectional associations between baseline participant characteristics and SPR are presented in Table 3. In univariate analyses, the following were associated $(p<0.05)$ with higher SPR: shorter self-reported height; lower alcohol consumption, physical activity and educational attainment; current use of AOM and calcium supplements; longer time since menopause; greater number of falls in the previous 12 months; history of fracture since aged 45 years; family history of hip fracture; higher FRAX scores for MOF and hip fracture; and increased comorbidity. Apart from associations regarding self-reported height and alcohol consumption, all were significant $(p<0.05)$ in mutually-adjusted analysis (FRAX variables were not included in the mutuallyadjusted model); however, the direction was reversed for time since menopause such that greater time was associated with reduced SPR.

In sensitivity analyses among participants with osteoporosis, currently taking AOM and with either of these conditions, many associations were not significant, perhaps due to the reduction in sample size. However, the following characteristics associated with SPR in the main analysis were also significant $(p<0.05)$ or had a trend towards significance $(p \leq 0.071)$ in sensitivity analyses (Supplementary Table S1): physical activity; currently taking calcium; and having a fracture since aged 45 years.

\section{Cluster Analysis of Participant Characteristics}

The four-cluster solution was optimal according to the BIC criterion; the number of participants in each cluster ranged from 459 to 904 .

Descriptive statistics for the participant characteristics according to each cluster are shown in Table 4. Compared to the other clusters, Cluster 1 had a greater proportion of women with the following characteristics: current use of $\operatorname{AOM}(35.4 \%$ vs $\leq 4.5 \%$ in other clusters), calcium supplements $(97.3 \%$ vs $\leq 1.1 \%)$ and Vitamin D supplements $(51.5 \%$ vs $\leq 13.1 \%)$; and a fracture since age 45 years $(33.7 \%$ vs $\leq 27.5 \%)$. Although not used in the cluster analysis algorithm, the proportion with higher SPR was also much higher in Cluster 1 (32.9\%) compared to other clusters $(\leq 11.2 \%)$.

The silhouette coefficient of 0.1 indicated that the clustering was not substantial. However, the results show that higher SPR and the risk factors for this variable tend to cluster together. 
Table 4 Participant characteristics according to each cluster

\begin{tabular}{|c|c|c|c|c|}
\hline Participant characteristic & Cluster1 $(n=489)$ & Cluster2 $(n=904)$ & Cluster3 $(n=730)$ & Cluster4 $(n=459)$ \\
\hline Age (years)* & $69.3(8.6)$ & $65.1(4.9)$ & $76.0(7.0)$ & $59.1(2.9)$ \\
\hline Self-reported height $(\mathrm{cm})^{*}$ & $161.7(6.8)$ & $163.0(6.3)$ & $160.3(6.5)$ & $163.7(6.0)$ \\
\hline Self-reported weight $(\mathrm{kg})^{*}$ & $64.8(10.9)$ & $70.0(12.7)$ & $68.4(13.2)$ & $69.0(13.0)$ \\
\hline $\operatorname{BMI}\left(\mathrm{kg} / \mathrm{m}^{2}\right)^{*}$ & $24.8(4.0)$ & $26.4(4.6)$ & $26.6(5.0)$ & $25.7(4.7)$ \\
\hline \multicolumn{5}{|l|}{ Self-perceived fracture risk } \\
\hline Lower & $96(19.6 \%)$ & $284(31.4 \%)$ & $217(29.7 \%)$ & $149(32.5 \%)$ \\
\hline Similar & $232(47.4 \%)$ & $538(59.5 \%)$ & $431(59.0 \%)$ & $276(60.1 \%)$ \\
\hline Higher & $161(32.9 \%)$ & $82(9.1 \%)$ & $82(11.2 \%)$ & $34(7.4 \%)$ \\
\hline Current smoker & $22(4.5 \%)$ & $59(6.5 \%)$ & $41(5.6 \%)$ & $27(5.9 \%)$ \\
\hline \multicolumn{5}{|l|}{ Alcohol consumption } \\
\hline None & $131(26.8 \%)$ & $212(23.5 \%)$ & $322(44.1 \%)$ & $84(18.3 \%)$ \\
\hline $1-6$ & $197(40.3 \%)$ & $410(45.4 \%)$ & $285(39.0 \%)$ & $195(42.5 \%)$ \\
\hline $7-13$ & $129(26.4 \%)$ & $205(22.7 \%)$ & $96(13.2 \%)$ & $131(28.5 \%)$ \\
\hline $14-20$ & $30(6.1 \%)$ & $66(7.3 \%)$ & $23(3.2 \%)$ & $41(8.9 \%)$ \\
\hline$>20$ & $2(0.4 \%)$ & $11(1.2 \%)$ & $4(0.5 \%)$ & $8(1.7 \%)$ \\
\hline \multicolumn{5}{|c|}{ Physically active compared to others } \\
\hline Not at all & $19(3.9 \%)$ & $12(1.3 \%)$ & $31(4.2 \%)$ & $9(2.0 \%)$ \\
\hline A little & $75(15.3 \%)$ & $113(12.5 \%)$ & $173(23.7 \%)$ & $68(14.8 \%)$ \\
\hline Somewhat & $257(52.6 \%)$ & $495(54.8 \%)$ & $322(44.1 \%)$ & $233(50.8 \%)$ \\
\hline Very & $138(28.2 \%)$ & $284(31.4 \%)$ & $204(27.9 \%)$ & $149(32.5 \%)$ \\
\hline \multicolumn{5}{|l|}{ Educational attainment } \\
\hline Below GCSE & $156(31.9 \%)$ & $222(24.6 \%)$ & $427(58.5 \%)$ & $84(18.3 \%)$ \\
\hline GCSE & $158(32.3 \%)$ & $356(39.4 \%)$ & $164(22.5 \%)$ & $169(36.8 \%)$ \\
\hline A-level & $80(16.4 \%)$ & $142(15.7 \%)$ & $81(11.1 \%)$ & $72(15.7 \%)$ \\
\hline Degree & $95(19.4 \%)$ & $184(20.4 \%)$ & $58(7.9 \%)$ & $134(29.2 \%)$ \\
\hline Current use of AOM & $173(35.4 \%)$ & $16(1.8 \%)$ & $33(4.5 \%)$ & $4(0.9 \%)$ \\
\hline Ever used oestrogen/HRT & $185(37.8 \%)$ & $472(52.2 \%)$ & $111(15.2 \%)$ & $176(38.3 \%)$ \\
\hline Currently taking calcium & $476(97.3 \%)$ & $0(0.0 \%)$ & $8(1.1 \%)$ & $0(0.0 \%)$ \\
\hline Currently taking Vit D & $252(51.5 \%)$ & $118(13.1 \%)$ & $71(9.7 \%)$ & $57(12.4 \%)$ \\
\hline \multicolumn{5}{|l|}{ Years since menopause } \\
\hline Less than 10 years & $71(14.5 \%)$ & $0(0.0 \%)$ & $0(0.0 \%)$ & $459(100.0 \%)$ \\
\hline $10-19$ years & $162(33.1 \%)$ & $685(75.8 \%)$ & $12(1.6 \%)$ & $0(0.0 \%)$ \\
\hline $20-29$ years & $141(28.8 \%)$ & $217(24.0 \%)$ & $326(44.7 \%)$ & $0(0.0 \%)$ \\
\hline 30 or more years & $115(23.5 \%)$ & $2(0.2 \%)$ & $392(53.7 \%)$ & $0(0.0 \%)$ \\
\hline \multicolumn{5}{|l|}{ Falls in previous 12 months } \\
\hline None & $288(58.9 \%)$ & $608(67.3 \%)$ & $435(59.6 \%)$ & $321(69.9 \%)$ \\
\hline Once & $125(25.6 \%)$ & $206(22.8 \%)$ & $185(25.3 \%)$ & $78(17.0 \%)$ \\
\hline 2 times or more & $76(15.5 \%)$ & $90(10.0 \%)$ & $110(15.1 \%)$ & $60(13.1 \%)$ \\
\hline Fracture since 45 years & $165(33.7 \%)$ & $90(10.0 \%)$ & $201(27.5 \%)$ & $31(6.8 \%)$ \\
\hline Family history of hip fracture & $84(17.2 \%)$ & $130(14.4 \%)$ & $83(11.4 \%)$ & $74(16.1 \%)$ \\
\hline \multicolumn{5}{|l|}{ Number of comorbidities } \\
\hline 0 & $124(25.4 \%)$ & $271(30.0 \%)$ & $100(13.7 \%)$ & $167(36.4 \%)$ \\
\hline 1 & $144(29.4 \%)$ & $319(35.3 \%)$ & $171(23.4 \%)$ & $152(33.1 \%)$ \\
\hline 2 & $110(22.5 \%)$ & $207(22.9 \%)$ & $229(31.4 \%)$ & $93(20.3 \%)$ \\
\hline 3 & $72(14.7 \%)$ & $88(9.7 \%)$ & $127(17.4 \%)$ & $32(7.0 \%)$ \\
\hline $4+$ & $39(8.0 \%)$ & $19(2.1 \%)$ & $103(14.1 \%)$ & $15(3.3 \%)$ \\
\hline
\end{tabular}

*Mean (SD)

The cluster analysis was restricted to participants with complete data for all variables that were used in the cluster analysis algorithm $(n=2582)$ BMI was derived from self-reported height and weight 
Table 5 Standard deviation difference in mean DXA aBMD parameters $(95 \% \mathrm{CI})$ per higher band of self-perceived fracture risk at baseline

\begin{tabular}{|c|c|c|c|c|}
\hline \multirow[t]{2}{*}{ Parameter } & \multicolumn{2}{|l|}{ Unadjusted } & \multicolumn{2}{|l|}{ Adjusted* } \\
\hline & Estimate $(95 \% \mathrm{CI})$ & $p$ & Estimate (95\%CI) & $p$ \\
\hline Whole body total & $-0.09(-0.21,0.03)$ & 0.143 & $-0.09(-0.22,0.04)$ & 0.172 \\
\hline Total hip & $-0.16(-0.26,-0.05)$ & 0.005 & $-0.11(-0.22,0.00)$ & 0.058 \\
\hline Femoral neck & $-0.18(-0.29,-0.08)$ & 0.001 & $-0.18(-0.29,-0.06)$ & 0.003 \\
\hline Total lumbar spine & $-0.13(-0.24,-0.02)$ & 0.018 & $-0.12(-0.24,0.00)$ & 0.053 \\
\hline
\end{tabular}

Self-perceived fracture risk was used as an ordinal variable with the following bands: 'much lower'; 'a little lower'; 'about the same'; 'a little higher'; and 'much higher'

Significant associations $(p<0.05)$ are highlighted in bold

$D X A$ dual-energy X-ray absorptiometry, $p p$ value, $a B M D$ areal bone mineral density

*Adjusted for age at time of DXA scan, follow-up time, height, weight-for-height residual, physical activity, smoking status, alcohol consumption, education, time since last menstrual cycle, use of anti-osteoporosis medication, calcium and vitamin D supplements, and oestrogen/hormone replacement therapy (pill/skin patch)

\section{Associations Between SPR and DXA aBMD Parameters}

The relationships between SPR and DXA aBMD parameters are presented in Table 5. Higher SPR was associated $(p<0.02)$ with subsequent lower aBMD of the total hip, femoral neck and total lumbar spine in unadjusted analysis; the association regarding femoral neck aBMD was robust to adjustment $(p=0.003)$, whereas for total hip it was reduced by around $30 \%$. The total hip encompasses the whole of the proximal femur region. In these women, it may be that the adjustment for body size and weight would have a much greater effect on this region of interest than on the femoral neck, which is a defined ROI-size not determined by the size of the bone. Also, whilst the total hip was reduced by $30 \%$, the difference remains, albeit of borderline significance using the arbitrary $p<0.05$ as the cut-off $(p=0.058)$.

\section{Associations Between SPR and Radial HRpQCT Parameters}

The associations between SPR and radial HRpQCT parameters are presented in Table 6. Higher SPR was associated with lower trabecular volumetric density and number, and higher trabecular separation in unadjusted and adjusted analysis $(p<0.03)$.

\section{Associations Between SPR and Tibial HRpQCT Parameters}

The associations between SPR and tibial HRpQCT parameters are also presented in Table 6. Higher SPR was associated with lower cortical area and thickness as well as lower trabecular volumetric density and thickness in unadjusted analysis $(p<0.05)$; relationships for cortical area and trabecular volumetric density were robust in adjusted analysis $(p<0.04)$. Higher SPR was related to higher trabecular separation in adjusted analysis $(p=0.027)$ and associations before adjustment were borderline significant $(p=0.055)$. When additionally adjusted for total hip aBMD, no associations regarding radial or tibial HRpQCT parameters were robust.

\section{Sensitivity Analysis}

In this subgroup, 69 women had a fracture since age 45 years, 31 were using AOM, 63 had a family history of hip fracture, and 141 women had at least one of these characteristics. These groups have been identified as higher risk and this prior knowledge/experience is likely to increase their SPR score, and may have led to previous BMD testing. We were therefore interested to investigate the associations between higher SPR and aBMD and HRpQCT parameters in groups where participants with prior fracture, AOM use, family history of hip fracture and any of these three characteristics were excluded (data not shown). When each of these four sets of exclusions were applied, higher SPR was associated with lower femoral neck aBMD in unadjusted and adjusted analysis. When women on AOM at baseline were excluded, higher SPR remained associated with lower radial trabecular number and higher trabecular separation both before and after adjustments. When women with previous fractures were excluded, higher SPR remained associated with lower radial trabecular number and higher trabecular separation in adjusted analyses; relationships were borderline significant when those with a family history of hip fracture were excluded. Higher SPR was related to lower tibial trabecular volumetric density when women with family history of hip fracture were excluded; in the other sets of sensitivity analyses, no other associations regarding tibial parameters were robust in both unadjusted and adjusted 
Table 6 Standard deviation difference in mean HRpQCT parameters $(95 \% \mathrm{CI})$ per higher band of self-perceived fracture risk at baseline

\begin{tabular}{|c|c|c|c|c|}
\hline \multirow[t]{2}{*}{ HRpQCT parameter } & \multicolumn{2}{|l|}{ Unadjusted } & \multicolumn{2}{|l|}{ Adjusted* } \\
\hline & Estimate $(95 \% \mathrm{CI})$ & $p$ & Estimate $(95 \% \mathrm{CI})$ & $p$ \\
\hline \multicolumn{5}{|l|}{ Radius } \\
\hline Total area & $-0.03(-0.16,0.09)$ & 0.609 & $0.02(-0.11,0.15)$ & 0.783 \\
\hline Trabecular area & $-0.01(-0.13,0.12)$ & 0.933 & $0.04(-0.09,0.17)$ & 0.526 \\
\hline Cortical area & $-0.12(-0.24,0.01)$ & 0.068 & $-0.14(-0.27,0.00)$ & 0.047 \\
\hline Cortical thickness & $-0.09(-0.21,0.04)$ & 0.159 & $-0.12(-0.26,0.02)$ & 0.090 \\
\hline Cortical volumetric density & $-0.02(-0.14,0.11)$ & 0.767 & $-0.09(-0.23,0.04)$ & 0.172 \\
\hline Cortical porosity & $-0.09(-0.21,0.04)$ & 0.176 & $0.00(-0.14,0.14)$ & 0.997 \\
\hline Cortical pores diameter & $-0.03(-0.15,0.10)$ & 0.682 & $-0.01(-0.16,0.14)$ & 0.906 \\
\hline Trabecular volumetric density & $-0.16(-0.28,-0.04)$ & $\mathbf{0 . 0 1 0}$ & $-0.16(-0.31,-0.02)$ & $\mathbf{0 . 0 2 7}$ \\
\hline Trabecular number & $-0.18(-0.31,-0.06)$ & 0.004 & $-0.19(-0.33,-0.04)$ & 0.010 \\
\hline Trabecular thickness & $-0.04(-0.17,0.08)$ & 0.499 & $-0.05(-0.20,0.10)$ & 0.522 \\
\hline Trabecular separation & $0.18(0.06,0.30)$ & 0.004 & $0.18(0.04,0.33)$ & 0.011 \\
\hline \multicolumn{5}{|l|}{ Tibia } \\
\hline Total area & $-0.01(-0.12,0.10)$ & 0.852 & $0.00(-0.10,0.11)$ & 0.953 \\
\hline Trabecular area & $0.02(-0.09,0.13)$ & 0.745 & $0.03(-0.08,0.13)$ & 0.636 \\
\hline Cortical area & $-0.15(-0.25,-0.04)$ & 0.008 & $-0.12(-0.23,-0.01)$ & $\mathbf{0 . 0 3 8}$ \\
\hline Cortical thickness & $-0.13(-0.24,-0.03)$ & 0.015 & $-0.10(-0.21,0.02)$ & 0.093 \\
\hline Cortical volumetric density & $-0.06(-0.17,0.05)$ & 0.287 & $-0.07(-0.18,0.05)$ & 0.240 \\
\hline Cortical porosity & $0.00(-0.11,0.11)$ & 0.952 & $0.03(-0.10,0.15)$ & 0.682 \\
\hline Cortical pores diameter & $-0.01(-0.12,0.10)$ & 0.832 & $0.02(-0.11,0.14)$ & 0.791 \\
\hline Trabecular volumetric density & $-0.16(-0.27,-0.06)$ & 0.003 & $-0.14(-0.26,-0.01)$ & $\mathbf{0 . 0 3 6}$ \\
\hline Trabecular number & $-0.09(-0.20,0.02)$ & 0.109 & $-0.13(-0.26,-0.01)$ & $\mathbf{0 . 0 3 5}$ \\
\hline Trabecular thickness & $-0.11(-0.22,-0.01)$ & 0.040 & $-0.03(-0.15,0.10)$ & 0.688 \\
\hline Trabecular separation & $0.11(0.00,0.22)$ & 0.055 & $0.14(0.02,0.26)$ & $\mathbf{0 . 0 2 7}$ \\
\hline
\end{tabular}

Self-perceived fracture risk was used as an ordinal variable with the following bands: 'much lower'; 'a little lower'; 'about the same'; 'a little higher'; and 'much higher'

Significant associations $(p<0.05)$ are highlighted in bold

$p p$ value; HRpQCT high resolution peripheral quantitative computed tomography

*Adjusted for age at time of HRpQCT scan, follow-up time, height, weight-for-height residual, physical activity, smoking status, alcohol consumption, education, time since last menstrual cycle, use of anti-osteoporosis medication, calcium and vitamin D supplements, and oestrogen/hormone replacement therapy (pill/ skin patch) analysis. When all three exclusions were applied, no tibial or radial associations were robust.

\section{Discussion}

In this study, we have identified personal characteristics associated with self-perception of risk of fracture. A cluster analysis of baseline participant characteristics identified one cluster, in which higher SPR, prior history of fracture since age of 45 , current use of AOM, vitamin D and calcium supplementation clustered together. Hence this seems to identify women who, through prior fracture experience, have initiated and remained on therapy and acknowledge their higher fracture risk. However, despite greater use of anti-osteoporosis medications, a higher SPR was still related to impaired bone density and microarchitecture measured a median of 7.5 years later. Associations were similar even when separately excluding the following groups of participants: previously experienced a fracture since age 45; reported a family history of hip fracture; and taking AOM. Although associations regarding tibial and radial HRpQCT parameters were attenuated when participants with any of these three characteristics were excluded, this could have been due to the reduction in sample size and robust associations between higher SPR and lower femoral neck aBMD remained after these exclusions.

To our knowledge this is the first time that associations between SPR and DXA aBMD and HRpQCT parameters among postmenopausal women have been examined, and suggests that women can correctly identify personal factors associated with heightened osteoporosis risk, but despite uptake of AOM, that risk remains elevated at around 7.5 years later. Findings from this study demonstrated that 
higher SPR bands are related to a decrease in areal BMD at the femoral neck and lower tibial trabecular volumetric density. There is evidence to suggest that a 1SD decrease in BMD is associated with a 1.5-threefold times higher fracture risk [13]. Our data suggests that it is likely that there will be increased fracture risk in women with higher SPR as they continue to lose bone and age.

There are limitations to our study. These are observational data that demonstrate associations, but not causality, and need to be tested in other populations. Secondly, the SPR questionnaire has not been validated. Finally, there is no information available if the participants had a DXA scan performed prior. Women who have had a prior fracture or took bone-specific treatment may well have had a DXA scan. It would not be unexpected that those participants rated their SPR as higher compared to other women of the same age. Those women were likely to integrate the bone protective behaviour and measures into their daily life resulting in a 'self-fulfilling prophesy'. However, even if it is taken into account, we still observed lower aBMD and less favourable HRpQCT parameters around 7 years later in this group. In many ways, this group represent the 'best case' scenario of osteoporosis care in that women have been identified as osteoporotic, recognise this diagnosis and remain on therapy to counteract this risk. The situation in many clinical cases may be much worse. Longer follow up of this group could be highly beneficial.

In the current study, the higher SPR was associated with higher FRAX scores for MOF and hip fracture. However, SPR of osteoporosis and osteoporotic fractures has been reported to be underestimated in postmenopausal women worldwide. Rothmann et al. observed that women participating in the Risk-Stratified Osteoporosis Strategy Evaluation (ROSE) study underestimated their fracture risk compared to the risk estimated by FRAX [14]. Similarly, findings from GLOW showed that women at increased fracture risk generally perceive their risk to be lower or about the same as women of the same age $[8,15]$. Furthermore, it was previously demonstrated in GLOW that SPR of fracture does capture some aspect of fracture risk not currently measured using the conventional fracture prediction tool FRAX [5]. The perception of personal risk has been shown to modify an individual's behaviour related to their bone health [5, 7]. Heightened self-perceived risks of osteoporosis and fracture significantly increases the likelihood of seeking medical advice hence, increasing the chances, in appropriate individuals, of being given a diagnosis of osteoporosis-a well known predictor of treatment initiation [7]. Moreover, heightened self-perceived risks of fracture are known to be associated with BMD testing.

Although the positive effect of risk perception on BMD testing has been previously described, the analysis of the relationship between the results of bone microarchitecture parameters and fracture risk perception is novel. There is evidence that other factors independent of aBMD, including skeletal properties of trabecular microstructure examined by HRpQCT, contribute to fracture risk [16-18]. This study suggests that there is an association between SPR and bone microarchitecture. Taking osteoporosis medications was strongly associated with a higher self-perceived fracture risk in this study. This concurs with findings from a crosssectional analysis of GLOW where women with higher SPR were more likely to report AOM use than women with lower SPR [5]. Barcenilla-Wong et al. also reported that elevated self-perceived risk of fracture increases the likelihood of taking AOM prospectively [7].

In conclusion, we have identified individual characteristics correlated with higher SPR, considered how they cluster together and studied relationships between SPR and subsequent objectively assessed bone health. This is particularly notable as previous research has suggested that while women often underestimate fracture risk, a higher SPR is associated with health seeking behaviour and better compliance with OP medication, as we observed in this study. An exploration of SPR through further studies, including qualitative work, may allow development of novel fracture prediction methods, and strategies to reduce fracture risk.

Funding Funding was supported by Arthritis Research UK [Grant No. 20380].

\section{Compliance with Ethical Standards}

Conflict of interest AE Litwic, LD Westbury, S Carter and KA Ward declare that they have no conflict of interest. C Cooper reports personal fees from Alliance for Better Bone Health, Amgen, Eli Lilly, GSK, Medtronic, Merck, Novartis, Pfizer, Roche, Servier, Takeda and UCB. EM Dennison reports consulting and speaker fees from Pfizer and UCB.

Human and Animal Rights and Informed Consent This research was performed in accordance with the Declaration of Helsinki and was approved by the UK Health Research Authority, reference 07/ MRE01/30. All participants gave informed consent.

Open Access This article is licensed under a Creative Commons Attribution 4.0 International License, which permits use, sharing, adaptation, distribution and reproduction in any medium or format, as long as you give appropriate credit to the original author(s) and the source, provide a link to the Creative Commons licence, and indicate if changes were made. The images or other third party material in this article are included in the article's Creative Commons licence, unless indicated otherwise in a credit line to the material. If material is not included in the article's Creative Commons licence and your intended use is not permitted by statutory regulation or exceeds the permitted use, you will need to obtain permission directly from the copyright holder. To view a copy of this licence, visit http://creativecommons.org/licenses/by/4.0/. 


\section{References}

1. Johnell O, Kanis JA (2006) An estimate of the worldwide prevalence and disability associated with osteoporotic fractures. Osteoporosis Int 17:1726-1733

2. van Staa TP, Dennison EM, Leufkens HG, Cooper C (2001) Epidemiology of fractures in England and Wales. Bone 29:517-522

3. Hernlund E, Svedbom A, Ivergard M, Compston J, Cooper C, Stenmark J, McCloskey EV, Jonsson B, Kanis JA (2013) Osteoporosis in the European Union: medical management, epidemiology and economic burden. A report prepared in collaboration with the International Osteoporosis Foundation (IOF) and the European Federation of Pharmaceutical Industry Associations (EFPIA). Arch Osteoporos 8:136

4. Burge R, Dawson-Hughes B, Solomon DH, Wong JB, King A, Tosteson A (2007) Incidence and economic burden of osteoporosis-related fractures in the United States, 2005-2025. JBMR 22:465-475

5. Litwic A, Compston J, Wyman A, Siris E, Gehlbach S, Adachi J, Chapurlat R, Díez-Pérez A, LaCroix A, Nieves J (2017) Selfperception of fracture risk: what can it tell us? Osteoporosis Int 28:3495-3500

6. Langer FW, da Silveira Codevilla AA, Bringhenti R, Dal Osto LC, Campos TRS, Martins TT, Barin AE, Rigo PH, Boufleuer ND, Santinon SF (2016) Low self-awareness of osteoporosis and fracture risk among postmenopausal women. Arch Osteoporos $11: 27$

7. Barcenilla-Wong AL, Chen JS, March LM (2014) Concern and risk perception: effects on osteoprotective behaviour. J Osteoporos 2014:142546

8. Gregson CL, Dennison EM, Compston JE et al (2014) Diseasespecific perception of fracture risk and incident fracture rates: GLOW cohort study. Osteoporosis Int 25:85-95

9. Hooven FH, Adachi JD, Adami S, Boonen S, Compston J, Cooper C, Delmas P, Diez-Perez A, Gehlbach S, Greenspan SL (2009) The global longitudinal study of Osteoporosis in Women (GLOW): rationale and study design. Osteoporos Int 20:1107-1116

10. Boutroy S, Bouxsein ML, Munoz F, Delmas PD (2005) In vivo assessment of trabecular bone microarchitecture by high-resolution peripheral quantitative computed tomography. J Clin Endocrinol Metab 90:6508-6515
11. Burghardt AJ, Kazakia GJ, Ramachandran S, Link TM, Majumdar $S$ (2010) Age- and gender-related differences in the geometric properties and biomechanical significance of intracortical porosity in the distal radius and tibia. JBMR 25:983-993

12. SPSS Inc (2001) The SPSS TwoStep cluster component: a scalable component enabling more efficient customer segmentation. Technical report. SPSS, Chicago, IL. Cited 18/09/2019. Available from https://www.spss.ch/upload/1122644952_The\%20SPSS\%20 TwoStep\%20Cluster\%20Component.pdf

13. Marshall D, Johnell O, Wedel H (1996) Meta-analysis of how well measures of bone mineral density predict occurrence of osteoporotic fractures. BMJ 312:1254

14. Rothmann MJ, Ammentorp J, Bech M, Gram J, Rasmussen OW, Barkmann R, Gluer CC, Hermann AP (2015) Self-perceived facture risk: factors underlying women's perception of risk for osteoporotic fractures: the Risk-Stratified Osteoporosis Strategy Evaluation study (ROSE). Osteoporosis Int 26:689-697

15. Siris ES, Gehlbach S, Adachi JD et al (2011) Failure to perceive increased risk of fracture in women 55 years and older: the Global Longitudinal Study of Osteoporosis in Women (GLOW). Osteoporosis Int 22:27-35

16. Edwards MH, Robinson DE, Ward KA, Javaid MK, WalkerBone K, Cooper C, Dennison EM (2016) Cluster analysis of bone microarchitecture from high resolution peripheral quantitative computed tomography demonstrates two separate phenotypes associated with high fracture risk in men and women. Bone 88:131-137

17. Litwic AE, Westbury LD, Robinson DE, Ward KA, Cooper C, Dennison EM (2017) Bone phenotype assessed by HRpQCT and Associations with Fracture Risk in the GLOW Study. Calcif Tissue Int 102(1):14-22

18. Samelson EJ, Broe KE, Xu H et al (2019) Cortical and trabecular bone microarchitecture as an independent predictor of incident fracture risk in older women and men in the Bone Microarchitecture International Consortium (BoMIC): a prospective study. Lancet Diabetes Endocrinol 7:34-43

Publisher's Note Springer Nature remains neutral with regard to jurisdictional claims in published maps and institutional affiliations. 